This item was submitted to Loughborough's Research Repository by the author.

Items in Figshare are protected by copyright, with all rights reserved, unless otherwise indicated.

\title{
The interaction of waves with horizontal cylinders in two-layer fluids
}

PLEASE CITE THE PUBLISHED VERSION

PUBLISHER

(c) Cambridge University Press

VERSION

VoR (Version of Record)

LICENCE

CC BY-NC-ND 4.0

\section{REPOSITORY RECORD}

Linton, C.M., and M. Mclver. 2019. "The Interaction of Waves with Horizontal Cylinders in Two-layer Fluids". figshare. https://hdl.handle.net/2134/4269. 
This item was submitted to Loughborough's Institutional Repository (https://dspace.lboro.ac.uk/) by the author and is made available under the following Creative Commons Licence conditions.

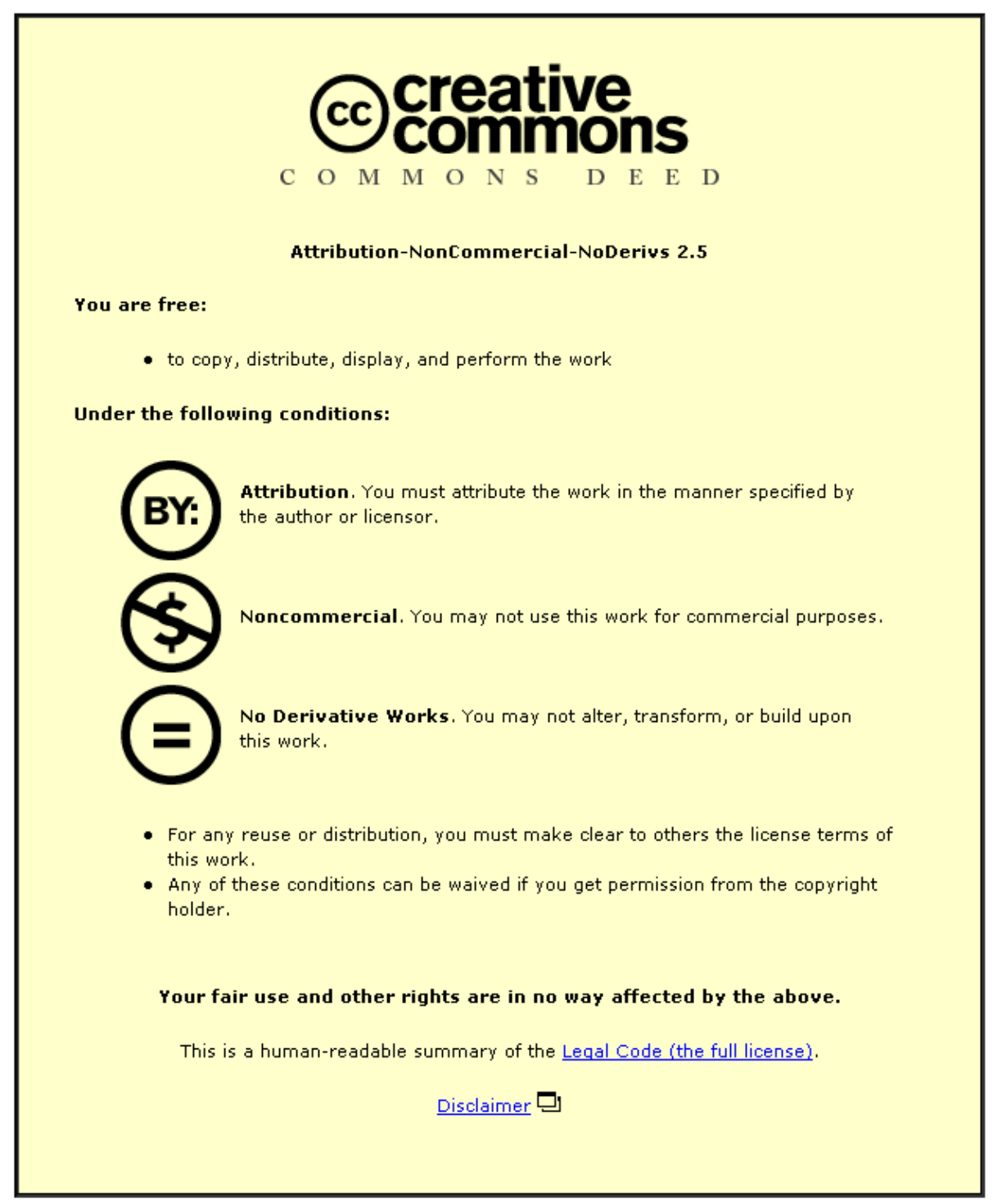

For the full text of this licence, please go to: http://creativecommons.org/licenses/by-nc-nd/2.5/ 


\title{
The interaction of waves with horizontal cylinders in two-layer fluids
}

\author{
By C. M. LINTON ANd M. McIVER \\ Department of Mathematical Sciences, Loughborough University of Technology, \\ Leicestershire LE11 3TU, UK
}

(Received 15 May 1995)

We consider two-dimensional problems based on linear water wave theory concerning the interaction of waves with horizontal cylinders in a fluid consisting of a layer of finite depth bounded above by a free surface and below by an infinite layer of fluid of greater density. For such a situation time-harmonic waves can propagate with two different wavenumbers $K$ and $k$. In a single-layer fluid there are a number of reciprocity relations that exist connecting the various hydrodynamic quantities that arise. These relations are systematically extended to the two-fluid case. It is shown that for symmetric bodies the solutions to scattering problems where the incident wave has wavenumber $K$ and those where it has wavenumber $k$ are related so that the solution to both can be found by just solving one of them. The particular problems of wave scattering by a horizontal circular cylinder in either the upper or lower layer are then solved using multipole expansions.

\section{Introduction}

Linear water wave theory is a widely used technique for determining how a wave is diffracted by a fixed or floating structure. The underlying assumption of the theory is that the amplitudes of any waves or body motions are small compared to the other length scales in the problem. At first order, this means that it is only necessary to consider the diffraction of a wave of a single frequency and direction, as linear superposition yields the diffraction pattern for an irregular sea. Furthermore, the velocity potential may be split into a part which describes the scattering of waves by a fixed structure and a part which describes the radiation of waves by the body into otherwise calm water. The radiation potential may be further split into a number of sub-potentials, each of which corresponds to the body moving in a separate mode of motion. The resulting potentials may be solved for separately but they are not all independent and a series of reciprocity relations which connect various scattering and radiation quantities have been derived by many authors over the years. These relations may be obtained by applying Green's theorem to two different potentials and a systematic derivation of all the first-order reciprocity relations is given by Newman (1976). They yield important information about the hydrodynamic loading on a body and the scattered wave field, and form a valuable check on the accuracy of any numerical wave diffraction code.

The reciprocity relations which exist have been derived for single-layer fluids, both in two and three dimensions and for finite- and infinite-depth fluids. More recently, however, interest has been extended to bodies which are immersed in two-layer fluids, each fluid having a different density. One reason for this is the suggestion by Friis, 
Grue \& Palm (1991) that an underwater pipe bridge might be built across one of the Norwegian fjords. A fjord typically consists of a layer of fresh water about $10 \mathrm{~m}$ thick on top of a very deep body of salt water. A bridge would have a diameter large enough to hold a two-lane road (about $10 \mathrm{~m}$ ) and would enter the water at a gentle slope passing through the free surface, the middle section being $40 \mathrm{~m}$ or so below the free surface, (and therefore totally within the lower layer), before rising through the upper layer and breaking the free surface on the other side of the fjord. If such a bridge were to be built, it would clearly be important to determine the effect a wave field would have on it.

The propagation of waves in a two-layer fluid which does not contain any bodies was first investigated by Stokes (1847) and a description of some of the types of linear motion which can occur is given in Lamb (1932, Art. 231). Since then many authors have studied the purely internal wave problem in which the upper fluid is either unbounded or is bounded above by a rigid lid. In particular, much work has been done on internal solitary waves in two-layer fluids, pioneered by authors such as Keulegan (1953), Long (1956) and Benjamin (1967). In addition, steady two-layer flow over bottom topography has been widely investigated (see, for example, Baines 1984 and references therein). Less work has been done on the unsteady problem in which the upper surface of the upper fluid is free. In this situation, Lamb (1932, Art. 231) showed that there are two possible linear wave systems at a given frequency, each with different wavenumber. These different modes may be interpreted easily when the ratio of the densities of the fluids is small. One mode corresponds to an oscillation which is mainly confined to the upper fluid and which is almost the same as that which would be obtained if its lower surface were a rigid wall. The second mode may be thought of as an oscillation in the lower fluid which is almost the same as would occur if its upper surface were free. For an arbitrary but stable density ratio, there is the possibility that some of the energy may be transferred from one mode to another if the wave field interacts with a body and this is one area which is investigated in this work.

In $\$ 2$ the radiation and scattering problems for an arbitrary, two-dimensional configuration of horizontal cylinders in a two-layer fluid are considered. Following the approach of Newman (1976), all the first-order reciprocity relations for a twolayer fluid are systematically derived from Green's theorem. Some of the relations such as the symmetry of the added mass and damping matrices and the analogue of the Haskind relations have already been reported by Wu (1992) and Sturova (1994) respectively, but a systematic derivation and collation of all the possible relations seems desirable. In $\S 3$, the wave scattering by a single, horizontal, circular cylinder which is totally contained in the lower fluid is investigated. The reasons for considering this specific body are two-fold. First, from a physical viewpoint, a circular cylinder is a reasonable model of a pipe bridge. Secondly, it is well-known (Dean 1948) that there is zero reflection of waves of any frequency by a circular cylinder submerged in a single-layer fluid of infinite depth and it is interesting to investigate the corresponding situation in the two-layer fluid. The analysis is slightly more complicated for the twolayer fluid because there are two possible reflected waves of different wavenumbers to consider. However, by using a multipole expansion analogous to that used by Ursell (1950) in the single-layer fluid, we find that regardless of the wavenumber of the incident wave, there is no reflection of energy into either mode. The partition of energy between the transmitted wave modes is investigated numerically with the aid of the reciprocity relations derived in $\$ 2$. The analysis is repeated in $\$ 4$ for a circular cylinder which is contained in the upper fluid and reflection of waves is found to 


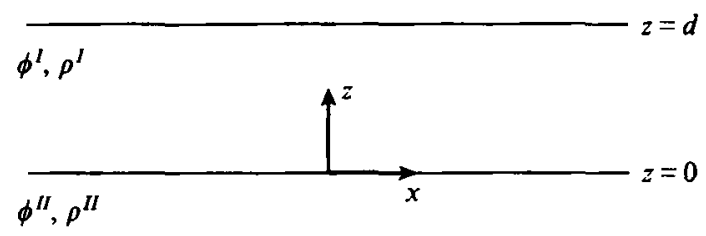

FIGURE 1. Definition sketch.

occur in this case. Finally, numerical calculations of the amount of energy reflected and transmitted in each mode are presented.

\section{Radiation and scattering problems in two-layer fluids}

The geometry of the two-layer fluid is shown in figure 1 and both fluids are assumed to be inviscid and incompressible. The horizontal coordinate is $x$ whilst the vertical coordinate is $z$ and is measured upwards from the undisturbed interface between the two fluids, the free surface being linearized about $z=d$. The upper fluid, $0<z<d$, will be referred to as region I, whilst the lower fluid, $z<0$, will be referred to as region II. The potential in the upper fluid (of density $\rho^{I}$ ) is $\phi^{I}$ and that in the lower fluid (of density $\rho^{I I}>\rho^{I}$ ) is $\phi^{I I}$. The motion is assumed to be irrotational and so both $\phi^{I}$ and $\phi^{I I}$ satisfy Laplace's equation:

$$
\nabla^{2} \phi^{I}=\nabla^{2} \phi^{\prime \prime}=0 .
$$

If we define $\rho=\rho^{I} / \rho^{I I}(<1)$ then the linearized boundary conditions on the interface and free surface are

$$
\begin{aligned}
\phi_{z}^{I} & =\phi_{z}^{I I} & & \text { on } z=0 \\
\rho\left(\phi_{z}^{I}-K \phi^{I}\right) & =\phi_{z}^{I I}-K \phi^{I I} & & \text { on } z=0, \\
\phi_{z}^{I} & =K \phi^{I} & & \text { on } z=d,
\end{aligned}
$$

where $K=\omega^{2} / \mathrm{g}$, the time-dependence of $\mathrm{e}^{-\mathrm{i} \omega t}$ having been suppressed. The boundary conditions (2.2) and (2.3) represent the continuity of normal velocity and pressure at the interface respectively.

In a two-layer fluid progressive waves take the form

$$
\begin{aligned}
\phi^{I} & =A \mathrm{e}^{\mathrm{tiux}}\left((u+K) \mathrm{e}^{u(z-d)}+(u-K) \mathrm{e}^{-u(z-d)}\right), \\
\phi^{I I} & =A \mathrm{e}^{ \pm i u x} \mathrm{e}^{u z}\left((u+K) \mathrm{e}^{-u d}-(u-K) \mathrm{e}^{u d}\right),
\end{aligned}
$$

where $u$ satisfies the dispersion relation

$$
(u-K)\left(K\left(\sigma+\mathrm{e}^{-2 u d}\right)-u\left(1-\mathrm{e}^{-2 u d}\right)\right)=0 .
$$

Here $\sigma=(1+\rho) /(1-\rho)$. It follows that either $u=K$ or $u=k$ where

$$
K\left(\sigma+\mathrm{e}^{-2 k d}\right)=k\left(1-\mathrm{e}^{-2 k d}\right) .
$$

This relation can be written in a number if different ways. Lamb (1932, Art. 231) gives the equivalent formula

$$
K(\rho+\operatorname{coth} k d)=k(1-\rho) .
$$

It is straightforward to show that the dispersion relation (2.5) has exactly one positive 
root $k$ which lies in the range

$$
K \sigma<k<\frac{K(\sigma+1)}{1-\mathrm{e}^{-2 K d \sigma}} .
$$

Waves of wavenumbers $K$ and $k$ can exist and they can propagate in either direction. In any wave radiation or scattering problem therefore, the far field will take the form of incoming and outgoing waves at each of the wavenumbers $K$ and $k$. It can be shown that the correct form for the far field is in general given by

$$
\begin{aligned}
\phi^{I} & \sim A^{ \pm} \mathrm{e}^{ \pm \mathrm{i} K x} \mathrm{e}^{K z}+B^{ \pm} \mathrm{e}^{ \pm \mathrm{i} k x} \mathrm{~g}(z)+C^{ \pm} \mathrm{e}^{\mp \mathrm{i} K x} \mathrm{e}^{K z}+D^{ \pm} \mathrm{e}^{\mp \mathrm{i} k x} g(z), \\
\phi^{I I} & \sim A^{ \pm} \mathrm{e}^{ \pm \mathrm{i} K x} \mathrm{e}^{K z}+B^{ \pm} \mathrm{e}^{ \pm \mathrm{i} k x} \mathrm{e}^{k z}+C^{ \pm} \mathrm{e}^{\mp i K x} \mathrm{e}^{K z}+D^{ \pm} \mathrm{e}^{\mp i k x} \mathrm{e}^{k z},
\end{aligned}
$$

as $x \rightarrow \pm \infty$, where

$$
\begin{aligned}
g(z) & =\frac{K \sigma-k}{K(\sigma-1)} \mathrm{e}^{k z}+\frac{K-k}{K(\sigma-1)} \mathrm{e}^{-k z} \\
& =\frac{\rho^{-1}}{1-\mathrm{e}^{2 k d}} \mathrm{e}^{k z}+\frac{\rho^{-1}-1+\mathrm{e}^{2 k d}}{1-\mathrm{e}^{2 k d}} \mathrm{e}^{-k z} .
\end{aligned}
$$

It may appear at first sight that there is a problem with (2.8) as $d \rightarrow \infty$ since the terms involving $\exp \{K z\}$ become large. However it should be remembered that these terms correspond to waves on the surface at $z=d$ and if the amplitudes of such waves are chosen to be unity a factor of $\exp \{-K d\}$ is introduced into the coefficients $A^{ \pm}, C^{ \pm}$. A convenient shorthand for (2.8) and (2.9) is

$$
\phi \sim\left\{A^{-}, B^{-}, C^{-}, D^{-} ; A^{+}, B^{+}, C^{+}, D^{+}\right\} .
$$

In the case of a single-layer fluid relations exist between the various hydrodynamic quantities that arise, which can be determined by an appropriate application of Green's theorem. These relations were discovered over a number of years and were systematically derived by Newman (1976). Similar results can be obtained in the two-fluid case.

We consider a situation in which there are a number of bodies, some in the upper layer, some in the lower layer, and some straddling the two. The boundaries of those bodies lying in the upper fluid will be denoted by $B_{I}$ and those in the lower fluid by $B_{I I}$. Assume that $\phi$ and $\psi$ are solutions to two different problems, with $\partial \phi / \partial n$ and $\partial \psi / \partial n$ given on the boundaries $B_{I}$ and $B_{I I}$, with the far-field form of $\phi$ given by (2.12), and

$$
\psi \sim\left\{\mathfrak{A}^{-}, \mathfrak{B}^{-}, \mathfrak{C}^{-}, \mathfrak{D}^{-} ; \mathfrak{A}^{+}, \mathfrak{B}^{+}, \mathfrak{C}^{+}, \mathfrak{D}^{+}\right\}
$$

We now apply Green's theorem, which for harmonic functions $\phi$ and $\psi$ takes the form

$$
\int_{S}\left(\phi \frac{\partial \psi}{\partial n}-\psi \frac{\partial \phi}{\partial n}\right) \mathrm{d} s=0
$$

where $S$ is the boundary of a fluid region completely contained in one of the fluid layers and $\partial / \partial n$ is the derivative with respect to the outward normal. Taking $S$ to be first the boundary of region I and then the boundary of region II, closed with lines at $x=-M$ and $x=L$, then using the result that on $z=0$, outside of any bodies,

$$
\rho\left(\phi^{I} \frac{\partial \psi^{I}}{\partial z}-\psi^{I} \frac{\partial \phi^{I}}{\partial z}\right)=\phi^{I I} \frac{\partial \psi^{I I}}{\partial z}-\psi^{I I} \frac{\partial \phi^{I I}}{\partial z}
$$

which follows from (2.2) and (2.3), we obtain after some lengthy algebra and letting 
$M, L \rightarrow \infty$,

$$
\begin{aligned}
& \int_{B_{l}} \rho\left(\phi^{I} \frac{\partial \psi^{I}}{\partial n}-\psi^{I} \frac{\partial \phi^{I}}{\partial n}\right) \mathrm{d} s \\
+ & \int_{B_{B_{I}}}\left(\phi^{I I} \frac{\partial \psi^{I I}}{\partial n}-\psi^{I I} \frac{\partial \phi^{I I}}{\partial n}\right) \mathrm{d} s=J_{K}\left(A^{+} \mathcal{C}^{+}-C^{+} \mathfrak{Q}^{+}+A^{-} \mathfrak{C}^{-}-C^{-} \mathfrak{U}^{-}\right) \\
+ & J_{k}\left(B^{+} \mathfrak{D}^{+}-D^{+} \mathfrak{B}^{+}+B^{-} \mathfrak{D}^{-}-D^{-} \mathfrak{B}^{-}\right)
\end{aligned}
$$

where

$$
\begin{aligned}
& J_{K}=\mathrm{i}\left(1+2 \rho K \int_{0}^{d} \mathrm{e}^{2 K z} \mathrm{~d} z\right), \\
& J_{k}=\mathrm{i}\left(1+2 \rho k \int_{0}^{d}[g(z)]^{2} \mathrm{~d} z\right) .
\end{aligned}
$$

In particular, if $\phi$ and $\psi$ are both scattering potentials having zero normal derivative on all body boundaries then the left-hand side of (2.16) is zero.

If we consider the scattering of waves by a fixed (but otherwise arbitrary) set of bodies, then in general there are four problems to consider. These are the scattering of an incident wave of wavenumber $K$ from $x=-\infty$, which we shall refer to as problem 1 ; the scattering of an incident wave of wavenumber $K$ from $x=+\infty$ (problem 2 ); the scattering of an incident wave of wavenumber $k$ from $x=-\infty$ (problem 3); and the scattering of an incident wave of wavenumber $k$ from $x=+\infty$ (problem 4). In each case there may be reflected and transmitted waves of wavenumbers $K$ and $k$. Capital letters $R$ and $T$ will be used to represent reflection and transmission coefficients corresponding to waves of wavenumber $K$ with $r$ and $t$ used for waves of wavenumber $k$. Thus the four problems are characterized using the notation of (2.12) by

$$
\begin{aligned}
& \phi_{1} \sim\left\{R_{1}, r_{1}, 1,0 ; T_{1}, t_{1}, 0,0\right\}, \\
& \phi_{2} \sim\left\{T_{2}, t_{2}, 0,0 ; R_{2}, r_{2}, 1,0\right\}, \\
& \phi_{3} \sim\left\{R_{3}, r_{3}, 0,1 ; T_{3}, t_{3}, 0,0\right\}, \\
& \phi_{4} \sim\left\{T_{4}, t_{4}, 0,0 ; R_{4}, r_{4}, 0,1\right\} .
\end{aligned}
$$

Applying (2.16) to $\phi_{1}$ and its complex conjugate $\overline{\phi_{1}}$ and writing $J=J_{k} / J_{K}$ leads to

$$
\left|R_{1}\right|^{2}+\left|T_{1}\right|^{2}+J\left(\left|r_{1}\right|^{2}+\left|t_{1}\right|^{2}\right)=1
$$

a result representing the conservation of energy. Similarly

$$
\begin{aligned}
& \left|R_{2}\right|^{2}+\left|T_{2}\right|^{2}+J\left(\left|r_{2}\right|^{2}+\left|t_{2}\right|^{2}\right)=1, \\
& \left|R_{3}\right|^{2}+\left|T_{3}\right|^{2}+J\left(\left|r_{3}\right|^{2}+\left|t_{3}\right|^{2}\right)=J, \\
& \left|R_{4}\right|^{2}+\left|T_{4}\right|^{2}+J\left(\left|r_{4}\right|^{2}+\left|t_{4}\right|^{2}\right)=J .
\end{aligned}
$$

Taking all possible pairs of functions from $\phi_{1}, \phi_{2}, \phi_{3}$ and $\phi_{4}$ and applying (2.16) leads to the relations

$$
\begin{aligned}
T_{1} & =T_{2}, \\
t_{3} & =t_{4}, \\
R_{3} & =J r_{1}, \\
R_{4} & =J r_{2},
\end{aligned}
$$




$$
\begin{aligned}
& T_{4}=J t_{1}, \\
& T_{3}=J t_{2} .
\end{aligned}
$$

The meaning of (2.27) and (2.28) is clear. The transmission coefficient for the wave of the same wavenumber as the incident wave is independent of the direction of incidence. This has a direct analogue in the non-stratified fluid case where it is well-known that the transmission coefficient is independent of the direction of the incident wave.

In order to shed light on the other relations it is convenient to define energies as follows:

$$
\begin{aligned}
& E_{j}^{R}=\left|R_{j}\right|^{2}, \quad E_{j}^{T}=\left|T_{j}\right|^{2}, \quad E_{j}^{r}=J\left|r_{j}\right|^{2}, \quad E_{j}^{t}=J\left|t_{j}\right|^{2}, \quad j=1,2, \\
& E_{j}^{R}=\left|R_{j}\right|^{2} / J, \quad E_{j}^{T}=\left|T_{j}\right|^{2} / J, \quad E_{j}^{r}=\left|r_{j}\right|^{2}, \quad E_{j}^{t}=\left|t_{j}\right|^{2}, \quad j=3,4 .
\end{aligned}
$$

Thus $E_{1}^{R}$ is the reflected energy at wavenumber $K$ due to an incident wave of unit energy and wavenumber $K$ from $x=-\infty$, and so on. The energy relations (2.23)-(2.26) then become

$$
E_{j}^{R}+E_{j}^{T}+E_{j}^{r}+E_{j}^{t}=1, \quad j=1,2,3 \text { or } 4 .
$$

In terms of these energies, equations (2.29)-(2.32) imply

$$
\begin{array}{ll}
E_{3}^{R}=E_{1}^{r}, & E_{4}^{R}=E_{2}^{r}, \\
E_{4}^{T}=E_{1}^{t}, & E_{3}^{T}=E_{2}^{t} .
\end{array}
$$

(Of course they also contain information about the phases of the various reflection and transmission coefficients.) In words the first of these results can be stated as: the energy reflected at wavenumber $K$ due to an incident wave from $x=-\infty(+\infty)$ of wavenumber $k$ is the same as the energy reflected at wavenumber $k$ due to an incident wave from $x=-\infty(+\infty)$ of wavenumber $K$. Equation (2.37) implies: the energy transmitted at wavenumber $K$ due to an incident wave from $x=+\infty(-\infty)$ of wavenumber $k$ is the same as the energy transmitted at wavenumber $k$ due to an incident wave from $x=-\infty(+\infty)$ of wavenumber $K$.

By applying (2.16) to one of the potentials $\phi_{j}, j=1,2,3$ or 4 , and the complex conjugate of a function corresponding to a different $j$ we obtain the following results:

$$
\begin{aligned}
& T_{1} \overline{R_{2}}+R_{1} \overline{T_{2}}=-J\left(t_{1} \overline{r_{2}}+r_{1} \overline{t_{2}}\right), \\
& T_{1} \overline{T_{3}}+R_{1} \overline{R_{3}}=-J\left(t_{1} \overline{t_{3}}+r_{1} \overline{r_{3}}\right), \\
& T_{1} \overline{R_{4}}+R_{1} \overline{T_{4}}=-J\left(t_{1} \overline{\bar{T}_{4}}+r_{1} \overline{t_{4}}\right), \\
& R_{2} \overline{T_{3}}+T_{2} \overline{R_{3}}=-J\left(r_{2} \overline{t_{3}}+t_{2} \overline{r_{3}}\right), \\
& R_{2} \overline{R_{4}}+T_{2} \overline{T_{4}}=-J\left(r_{2} \overline{r_{4}}+t_{2} \overline{t_{4}}\right), \\
& T_{3} \overline{R_{4}}+R_{3} \overline{T_{4}}=-J\left(t_{3} \overline{r_{4}}+r_{3} \overline{t_{4}}\right) .
\end{aligned}
$$

For the case of a body symmetric about $x=0$ the direction of the incident wave is immaterial, problems 2 and 4 being equivalent to problems 1 and 3 respectively. We thus need only consider problems 1 and 3 and a number of simplifications to the above equations are possible. First we note that all the relations stated previously reduce to the following eight equations:

$$
\begin{aligned}
\left|R_{1}\right|^{2}+\left|T_{1}\right|^{2}-1 & =-J\left(\left|r_{1}\right|^{2}+\left|t_{1}\right|^{2}\right), \\
\operatorname{Re}\left\{T_{1} \overline{R_{1}}\right\} & =-J \operatorname{Re}\left\{t_{1} \bar{r}_{1}\right\}, \\
\left|R_{3}\right|^{2}+\left|T_{3}\right|^{2} & =J\left(1-\left|r_{3}\right|^{2}-\left|t_{3}\right|^{2}\right),
\end{aligned}
$$




$$
\begin{aligned}
\operatorname{Re}\left\{T_{3} \overline{R_{3}}\right\} & =-J \operatorname{Re}\left\{t_{3} \overline{r_{3}}\right\}, \\
R_{3} & =J r_{1}, \\
T_{3} & =J t_{1}, \\
T_{1} \overline{T_{3}}+R_{1} \overline{R_{3}} & =-J\left(t_{1} \overline{t_{3}}+r_{1} \overline{r_{3}}\right), \\
T_{1} \overline{R_{3}}+R_{1} \overline{T_{3}} & =-J\left(t_{1} \overline{r_{3}}+r_{1} \overline{t_{3}}\right) .
\end{aligned}
$$

The first four equations, which are real, provide relations between the reflection and transmission coefficients for a particular problem, whereas equations (2.48)(2.51), which are complex, provide relationships between the solutions of problem 1 and those of problem 3. If we consider $R_{1}, T_{1}, r_{1}$, and $t_{1}$ as known then these equations constitute four complex equations for the four complex unknowns $R_{3}, T_{3}$, $r_{3}$, and $t_{3}$. In fact we obtain

$$
\begin{aligned}
& \overline{r_{3}}\left(t_{1}^{2}-r_{1}^{2}\right)=2 \mathrm{i} T_{1} \operatorname{Im}\left\{r_{1} \overline{t_{1}}\right\}+R_{1}\left(\left|r_{1}\right|^{2}-\left|t_{1}\right|^{2}\right), \\
& \overline{t_{3}}\left(t_{1}^{2}-r_{1}^{2}\right)=2 \mathrm{i} R_{1} \operatorname{Im}\left\{r_{1} \bar{t}_{1}\right\}+T_{1}\left(\left|r_{1}\right|^{2}-\left|t_{1}\right|^{2}\right) .
\end{aligned}
$$

Thus providing $r_{1} \neq \pm t_{1}$ equations (2.48), (2.49), (2.52) and (2.53) determine the solution to problem 3 once that of problem 1 is known.

There are a great many relationships that can be determined from equations (2.44)-(2.51); we simply note the following. From (2.44), (2.46), (2.48) and (2.49) we have

$$
E_{1}^{R}+E_{1}^{T}=E_{3}^{r}+E_{3}^{t} .
$$

In other words the energy that is scattered at wavenumber $K$ due to an incident wave of wavenumber $K$ is the same as the energy scattered at wavenumber $k$ due to an incident wave of wavenumber $k$. Equations (2.45) and (2.47), (2.48) and (2.49) imply that

$$
T_{1} \overline{R_{1}}+R_{1} \overline{T_{1}}=t_{3} \overline{r_{3}}+r_{3} \overline{t_{3}}
$$

and this and (2.54) are equivalent to the pair of equations

$$
\left|R_{1} \pm T_{1}\right|^{2}=\left|r_{3} \pm t_{3}\right|^{2}
$$

Up till now we have considered only scattering potentials. We will now return to the case of an arbitrary geometry and see what further relations can be obtained using radiation potentials and combinations of radiation and scattering potentials in (2.16).

Let $\phi_{\alpha}$ be the radiation potential due to a single body oscillating in such a way that

$$
\frac{\partial \phi_{\alpha}}{\partial n}=n_{\alpha} \quad \text { on } S_{B}
$$

where $n_{\alpha}$ is the component of the inward normal to the body in the direction $\alpha$ and $S_{B}$ is the body boundary, which for simplicity we will assume is entirely contained within region I or region II. We can characterize the far-field behaviour of $\phi_{\alpha}$ by

$$
\phi_{\alpha} \sim\left\{A^{-}, B^{-}, 0,0 ; A^{+}, B^{+}, 0,0\right\} .
$$

Let $\phi_{\beta}$ be a second radiation potential defined in a similar way with the far-field behaviour

$$
\phi_{\beta} \sim\left\{\mathfrak{A}^{-}, \mathfrak{B}^{-}, 0,0 ; \mathfrak{U}^{+}, \mathfrak{B}^{+}, 0,0\right\},
$$

then application of (2.16) to $\phi_{\alpha}$ and $\phi_{\beta}$, in the case when the body boundary is 
contained within region $\mathrm{I}$, yields

$$
-\mathrm{i} \omega \mu_{\alpha \beta}+v_{\alpha \beta} \equiv \mathrm{i} \omega \rho^{I} \int_{S_{B}} \phi_{\alpha} n_{\beta} \mathrm{d} s=\mathrm{i} \omega \rho^{I} \int_{S_{B}} \phi_{\beta} n_{\alpha} \mathrm{d} s=-\mathrm{i} \omega \mu_{\beta \alpha}+v_{\beta \alpha},
$$

where the real matrices $\mu$ and $v$ are respectively the added-mass and damping matrices. If the body boundary is contained within region II then $\rho^{I}$ must be replaced by $\rho^{I I}$ in these formulas. Thus (2.60) states that, as in the single-layer fluid case, both these matrices are symmetric.

From (2.59), the complex conjugate of $\phi_{\beta}$ has the far-field behaviour

$$
\overline{\phi_{\beta}} \sim\left\{0,0, \overline{\mathfrak{A}^{-}}, \overline{\mathfrak{B}^{-}} ; 0,0, \overline{\mathfrak{A}^{+}}, \overline{\mathfrak{B}^{+}}\right\},
$$

and so application of (2.16) to $\phi_{\alpha}$ and $\overline{\phi_{\beta}}$ yields

$$
\delta \int_{S_{B}}\left(\phi_{\alpha} n_{\beta}-\overline{\phi_{\beta}} n_{\alpha}\right) \mathrm{d} s=J_{K}\left(A^{+} \overline{\mathfrak{Q}^{+}}+A^{-} \overline{\mathfrak{Q}^{-}}\right)+J_{k}\left(B^{+} \overline{\mathfrak{B}^{+}}+B^{-} \overline{\mathfrak{B}^{-}}\right),
$$

where $\delta=\rho$ if the body is situated in the upper layer and $\delta=1$ if the body is situated in the lower layer. In particular if $\alpha=\beta$ then (2.62) becomes

$$
2 \mathrm{i} \delta \operatorname{Im} \int_{S_{B}} \phi_{\alpha} n_{\alpha} \mathrm{d} s=J_{K}\left(\left|A^{+}\right|^{2}+\left|A^{-}\right|^{2}\right)+J_{k}\left(\left|B^{+}\right|^{2}+\left|B^{-}\right|^{2}\right),
$$

showing that the diagonal elements of the damping matrix are proportional to the energy radiated by the oscillating body and, from (2.17) and (2.18), are non-negative. Again this is completely analogous to the single-layer fluid result.

Further results can be obtained by applying (2.16) to $\phi_{\alpha}$ and each of the scattering potentials characterized by (2.19)-(2.22) in turn. Using $\phi_{x}$ and $\phi_{1}$ gives rise to

$$
\delta \int_{S_{B}} \phi_{1} n_{\alpha} \mathrm{d} s=-J_{K} A^{-},
$$

which can be interpreted physically as meaning that the exciting force on the body in direction $\alpha$ due to a wave of wavenumber $K$ from $x=-\infty$ is proportional to the amplitude of the wave of wavenumber $K$ radiated to $-\infty$ by the body oscillating in direction $\alpha$. Similarly

$$
\begin{aligned}
& \delta \int_{S_{B}} \phi_{2} n_{\alpha} \mathrm{d} s=-J_{K} A^{+}, \\
& \delta \int_{S_{B}} \phi_{3} n_{\alpha} \mathrm{d} s=-J_{k} B^{-}, \\
& \delta \int_{S_{B}} \phi_{4} n_{\alpha} \mathrm{d} s=-J_{k} B^{+},
\end{aligned}
$$

each of which has a similar physical interpretation. Equations (2.64)-(2.67) are extensions to two-layer fluids of the Haskind relations, see Newman (1976).

Finally we can also obtain results by applying (2.16) to $\phi_{x}-\overline{\phi_{x}}$ and each of the scattering potentials in turn. The far-field behaviour of $\phi_{x}-\overline{\phi_{\alpha}}$ is characterized by

$$
\phi_{x}-\overline{\phi_{\alpha}} \sim\left\{A^{-}, B^{-},-\overline{A^{-}},-\overline{B^{-}} ; A^{+}, B^{+},-\overline{A^{+}},-\overline{B^{+}}\right\},
$$

and it follows from (2.57) that the normal derivative of $\phi_{\alpha}-\overline{\phi_{x}}$ is zero on the body. Hence we obtain

$$
A^{-}+T_{1} \overline{A^{+}}+R_{1} \overline{A^{-}}=-J\left(t_{1} \overline{B^{+}}+r_{1} \overline{B^{-}}\right),
$$




$$
\begin{aligned}
A^{+}+R_{2} \overline{A^{+}}+T_{2} \overline{A^{-}} & =-J\left(r_{2} \overline{B^{+}}+t_{2} \overline{B^{-}}\right), \\
T_{3} \overline{A^{+}}+R_{3} \overline{A^{-}} & =-J\left(t_{3} \overline{B^{+}}+r_{3} \overline{B^{-}}+B^{-}\right), \\
R_{4} \overline{A^{+}}+T_{4} \overline{A^{-}} & =-J\left(r_{4} \overline{B^{+}}+t_{4} \overline{B^{-}}+B^{+}\right),
\end{aligned}
$$

which extend the Newman relations to the two-layer fluid case.

\section{Incident wave expansions}

In problem 1 the form of the incident wave is

$$
\phi_{i n c}=\mathrm{e}^{\mathrm{i} K x} \mathrm{e}^{K z}
$$

whereas in problem 3 we have

$$
\phi_{i n c}^{l}=\mathrm{e}^{\mathrm{i} k x} \mathrm{~g}(z), \quad \phi_{i n c}^{I I}=\mathrm{e}^{\mathrm{i} k x} \mathrm{e}^{k z} .
$$

If we define polar coordinates $r, \theta$ by

$$
x=r \sin \theta, \quad \zeta-z=r \cos \theta,
$$

then expansions of these incident wave potentials about $x=0, z=\zeta$ can be found using the results

$$
\begin{aligned}
& \mathrm{e}^{s(\zeta-z)} \mathrm{e}^{\mathrm{i} s x}=\sum_{m=0}^{\infty} \frac{(s r)^{m}}{m !} \mathrm{e}^{\mathrm{i} m \theta}, \\
& \mathrm{e}^{s(z-\zeta)} \mathrm{e}^{\mathrm{i} s x}=\sum_{m=0}^{\infty} \frac{(-s r)^{m}}{m !} \mathrm{e}^{-\mathrm{i} m \theta} .
\end{aligned}
$$

For the case of wavenumber $K$ we obtain

$$
\phi_{i n c}=\mathrm{e}^{K \zeta} \sum_{m=0}^{\infty} \frac{(-K r)^{m}}{m !} \mathrm{e}^{-\mathrm{i} m \theta}
$$

whereas in the case of an incident wave of wavenumber $k$ the form will be different depending on whether $\zeta>0$ or $\zeta<0$. In the latter case $\phi_{i n c}^{I I}$ is simply given by (2.78) with $K$ replaced by $k$. However if $\zeta>0$ we obtain

$$
\phi_{i n c}^{I}=\frac{(K \sigma-k) \mathrm{e}^{k \zeta}}{K(\sigma-1)} \sum_{m=0}^{\infty} \frac{(-k r)^{m}}{m !} \mathrm{e}^{-\mathrm{i} m \theta}+\frac{(K-k) \mathrm{e}^{-k \zeta}}{K(\sigma-1)} \sum_{m=0}^{\infty} \frac{(k r)^{m}}{m !} \mathrm{e}^{\mathrm{i} m \theta}
$$

\section{Scattering by a cylinder in the lower fluid}

The technique that will be employed is that of multipole expansions, used originally by Ursell (1950) to describe wave scattering by a cylinder submerged in a non-stratified fluid. In this context multipoles are singular solutions of the governing equation which satisfy all the boundary conditions of the problem except that on the cylinder. The idea of constructing multipoles for a two-layer fluid is not new: Kassem (1982) constructed such functions for a two-layer fluid bounded above and below by rigid walls. The method used below to construct multipoles for a finite fluid layer bounded above by a free surface and below by an infinite fluid layer of different density is essentially an extension of the method used by Thorne (1953) for the single-layer case.

For the case of a cylinder centred at $x=0, z=\zeta<0$ of radius $a(<|\zeta|)$ we need to develop multipoles singular at $z=\zeta, \zeta<0$. It is convenient to distinguish those 
multipoles symmetric about $x=0$ and those antisymmetric about this line. These will be denoted by $\phi_{n}^{s}$ and $\phi_{n}^{a}$ respectively. The form of these functions will be different in regions I and II and we will use the notation $\phi_{n}^{I s}$ when referring to the form of $\phi_{n}^{s}$ in region $I$, etc.

Solutions of Laplace's equation singular at $z=\zeta$ are $r^{-n} \cos n \theta$ and $r^{-n} \sin n \theta$, $n \geqslant 1$, and these have the integral representations, valid for $z>\zeta$,

$$
\begin{aligned}
\frac{\cos n \theta}{r^{n}} & =\frac{(-1)^{n}}{(n-1) !} \int_{0}^{\infty} u^{n-1} \mathrm{e}^{-u(z-\zeta)} \cos u x \mathrm{~d} u, \\
\frac{\sin n \theta}{r^{n}} & =\frac{(-1)^{n+1}}{(n-1) !} \int_{0}^{\infty} u^{n-1} \mathrm{e}^{-u(z-\zeta)} \sin u x \mathrm{~d} u
\end{aligned}
$$

(Gradshteyn \& Ryzhik 1965, 3.944(5) and (6)). It is then straightforward to add suitable solutions of Laplace's equation to these functions so that the boundary conditions (2.2)-(2.4) are satisfied. We obtain

$$
\begin{aligned}
\phi_{n}^{I s} & =\frac{(-1)^{n}}{(n-1) !} \oint_{0}^{\infty} u^{n-1}\left[A_{L}(u) \mathrm{e}^{u z}+B_{L}(u) \mathrm{e}^{-u z}\right] \cos u x \mathrm{~d} u \\
\phi_{n}^{I I s} & =\frac{\cos n \theta}{r^{n}}+\frac{(-1)^{n}}{(n-1) !} \oint_{0}^{\infty} u^{n-1} C_{L}(u) \mathrm{e}^{u z} \cos u x \mathrm{~d} u, \\
\phi_{n}^{I a} & =\frac{(-1)^{n+1}}{(n-1) !} \oint_{0}^{\infty} u^{n-1}\left[A_{L}(u) \mathrm{e}^{u z}+B_{L}(u) \mathrm{e}^{-u z}\right] \sin u x \mathrm{~d} u, \\
\phi_{n}^{I I a} & =\frac{\sin n \theta}{r^{n}}+\frac{(-1)^{n+1}}{(n-1) !} \oint_{0}^{\infty} u^{n-1} C_{L}(u) \mathrm{e}^{u z} \sin u x \mathrm{~d} u,
\end{aligned}
$$

where

$$
\begin{aligned}
& A_{L}(u)=K(1+\sigma)(u+K) \mathrm{e}^{u(\zeta-2 d)} /(u-K) h(u), \\
& B_{L}(u)=K(1+\sigma) \mathrm{e}^{u \zeta} / h(u), \\
& C_{L}(u)=(u+K) \mathrm{e}^{u \zeta}\left[(u+K \sigma) \mathrm{e}^{-2 u d}-u+K\right] /(u-K) h(u) .
\end{aligned}
$$

Here

$$
h(u)=(u+K) \mathrm{e}^{-2 u d}-u+K \sigma
$$

and from (2.5) is such that $h(k)=0$. The path of integration is indented to pass beneath the poles of the integrand at $u=K$ and $u=k$.

These multipoles can be expanded about $r=0$. We obtain, valid for $r<|\zeta|$,

$$
\begin{aligned}
& \phi_{n}^{s}=\frac{\cos n \theta}{r^{n}}+\sum_{m=0}^{\infty} A_{n m} r^{m} \cos m \theta, \\
& \phi_{n}^{a}=\frac{\sin n \theta}{r^{n}}+\sum_{m=1}^{\infty} A_{n m} r^{m} \sin m \theta,
\end{aligned}
$$

where

$$
A_{n m}=\frac{(-1)^{n+m}}{(n-1) ! m !} \oint_{0}^{\infty} u^{n+m-1} C_{L}(u) \mathrm{e}^{u \zeta} \mathrm{d} u .
$$

Note that $A_{n m}$ is the same for $\phi_{n}^{s}$ and $\phi_{n}^{a}$.

In order to find the behaviour of $\phi_{n}^{s}$ and $\phi_{n}^{a}$ as $|x| \rightarrow \infty$ it is clear from (2.8) and (2.9) that we only need to consider region II as this fully determines the coefficients 
$A^{ \pm}, B^{ \pm}, C^{ \pm}$and $D^{ \pm}$. From (3.4) and (3.6) it follows that as $x \rightarrow \pm \infty$

$$
\begin{aligned}
\phi_{n}^{I l s} & \sim \frac{(-1)^{n} \pi \mathrm{i}}{(n-1) !}\left[K^{n-1} R\left(C_{L}: K\right) \mathrm{e}^{ \pm \mathrm{i} K x} \mathrm{e}^{K z}+k^{n-1} R\left(C_{L}: k\right) \mathrm{e}^{ \pm \mathrm{ikx}} \mathrm{e}^{k z}\right], \\
\phi_{n}^{I I a} & \sim \mp \frac{(-1)^{n} \pi}{(n-1) !}\left[K^{n-1} R\left(C_{L}: K\right) \mathrm{e}^{ \pm \mathrm{i} K x} \mathrm{e}^{K z}+k^{n-1} R\left(C_{L}: k\right) \mathrm{e}^{ \pm \mathrm{i} k x} \mathrm{e}^{k z}\right],
\end{aligned}
$$

where $R\left(C_{L}: u_{0}\right)$ is the residue of $C_{L}(u)$ at $u=u_{0}$.

To solve the scattering problem we put

$$
\phi=\phi_{i n c}+\sum_{n=1}^{\infty} a^{n}\left(\alpha_{n} \phi_{n}^{a}+\beta_{n} \phi_{n}^{s}\right)
$$

where $\phi_{\text {inc }}$ can be given by either (2.73) or (2.74). Since $\partial \phi / \partial r=0$ on $r=a$ we have

$$
\sum_{n=1}^{\infty} a^{n} \frac{\partial}{\partial r}\left(\alpha_{n} \phi_{n}^{a}+\beta_{n} \phi_{n}^{s}\right)=-\frac{\partial \phi_{i n c}}{\partial r} \quad \text { on } r=a \text {. }
$$

Substituting the polar expansions of the multipoles (3.11) and (3.12) and of the incident wave, leads to uncoupled sets of equations for the unknowns $\alpha_{n}$ and $\beta_{n}$. Since the matrix is the same in each system and the right-hand sides of each system differ by a factor of $-i$ we find that

$$
\alpha_{n}=-\mathrm{i} \beta_{n}
$$

and in the case of an incident wave of wavenumber $K$ the $\beta_{n}$ satisfy the infinite system of equations

$$
\beta_{m}-\sum_{n=1}^{\infty} \beta_{n} a^{n+m} A_{n m}=\frac{(-K a)^{m}}{m !} \mathrm{e}^{K \zeta} \quad m=1,2, \ldots
$$

For an incident wave of wavenumber $k$ it is simply necessary to replace $K$ by $k$ in the right-hand side of this system of equations. These equations can be solved by truncation and the solution $\phi$ is then given by

$$
\phi=\phi_{i n c}+\sum_{n=1}^{\infty} a^{n} \beta_{n}\left(\phi_{n}^{s}-\mathrm{i} \phi_{n}^{a}\right) .
$$

It follows immediately from (3.14) and (3.15) that as $x \rightarrow-\infty$

$$
\phi \sim \phi_{\text {inc }}
$$

and hence that

On the other hand as $x \rightarrow+\infty$

$$
R_{1}=r_{1}=R_{3}=r_{3}=0 .
$$

$$
\phi^{I I} \sim \phi_{i n c}^{I I}+2 \pi \mathrm{i} \sum_{n=1}^{\infty} \frac{(-1)^{n} a^{n} \beta_{n}}{(n-1) !}\left[K^{n-1} R\left(C_{L}: K\right) \mathrm{e}^{\mathrm{i} K x} \mathrm{e}^{K z}+k^{n-1} R\left(C_{L}: k\right) \mathrm{e}^{\mathrm{i} k x} \mathrm{e}^{k z}\right]
$$

from which the transmission coefficients $T_{1}, t_{1}, T_{3}$ and $t_{3}$ are readily obtained.

In a single-layer fluid of infinite depth it is well known that the reflection coefficient for a submerged horizontal cylinder is zero. This was first discovered by Dean (1948). This is not the case however in finite depth (see, for example, Evans \& Linton 1989) and as we shall see it is not the case for a cylinder in the upper layer of a two-layer fluid. Crucial to the derivation of (3.22) was the fact that the coefficients $A_{n m}$ in the polar 
expansions of the symmetric and antisymmetric multipoles are the same. However as soon as it is necessary for the multipoles to satisfy boundary conditions both above and below the cylinder this equivalence is lost. The reflection coefficients for the problem under discussion here are functions of the four dimensionless parameters $K a, K|\zeta|, K d$ and $\sigma$ and it is remarkable that a function of four parameters should vanish identically. In fact preliminary investigations suggest that zero reflection occurs when any number of layers are situated above an infinitely deep body of fluid containing a circular cylinder.

Using (3.22) we see that for the case of a horizontal circular cylinder submerged in the lower layer of a two-layer fluid equations (2.44)-(2.51) reduce to

$$
\begin{aligned}
\left|T_{1}\right|^{2}-1 & =-J\left|t_{1}\right|^{2}, \\
\left|T_{3}\right|^{2} & =J\left(1-\left|t_{3}\right|^{2}\right), \\
T_{3} & =J t_{1}, \\
T_{1} \overline{T_{3}} & =-J t_{1} \overline{t_{3}},
\end{aligned}
$$

Provided $t_{1} \neq 0$ equations (3.26) and (3.27) can be used to determine $T_{3}$ and $t_{3}$ once $T_{1}$ and $t_{1}$ are known. Equations (3.24)-(3.26) can be combined to give

$$
E_{1}^{T}=E_{3}^{t}
$$

showing that the amount of the incident wave energy that is transmitted at the same wavenumber as the incident wave is independent of whether the incident wave has wavenumber $K$ or $k$. Finally equations (3.26) and (3.27) imply that

$$
\arg \left(T_{1}\right)-2 \arg \left(t_{1}\right)+\arg \left(t_{3}\right)=(2 n+1) \pi,
$$

where $n$ is an integer.

In order to compute solutions we must solve (3.19) using a truncation procedure. We thus convert this system into an $N \times N$ system of equations and check the convergence of the results as $N$ increases. As is typical with multipole methods the convergence is extremely rapid and a value of $N=6$ appears to give reflected and transmitted energies to five decimal places of accuracy. The results shown below were all computed using this value for $N$ and they were all checked against the conservation of energy condition (2.35). Also both problem 1 and problem 3 were solved and the results checked against the relations (3.26) and (3.27). All these relationships were satisfied to a high degree of numerical accuracy.

Figure 2 shows the proportion of the incident energy that is transmitted at wavenumbers $K$ and $k$ due to a wave of wavenumber $K$ incident on a cylinder in the lower layer. The geometrical parameters are chosen to be typical of a situation that might be found in the case of an underwater pipe bridge, namely $d / a=2$ and $\zeta / a=-2$. The ratio of the densities in the two layers is taken to be 0.97 , corresponding to the ratio of the densities of fresh and salt water. The value of $k a$, found by solving the dispersion relation (2.5) is shown along the top of the figure. It is clear that for these parameter values the amount of energy that is transferred from the incident wavenumber to wavenumber $k$ is very small indeed. These results are typical for the density ratio $\rho=0.97$. For smaller values of $\rho$ a greater effect is observed but the effect is still small. For example, with $d / a$ and $\zeta / a$ as in figure 2 but with $\rho=0.5$ the maximum value of $E_{1}^{t}$ is approximately 0.032 and occurs when $K a \approx 0.24(k a \approx 0.77)$.

If we let $\rho \rightarrow 0$ in this problem (corresponding to $\sigma \rightarrow 1$ ) then we see that the multipoles defined by (3.4) and (3.6) go over to the single-layer multipoles for infinite 


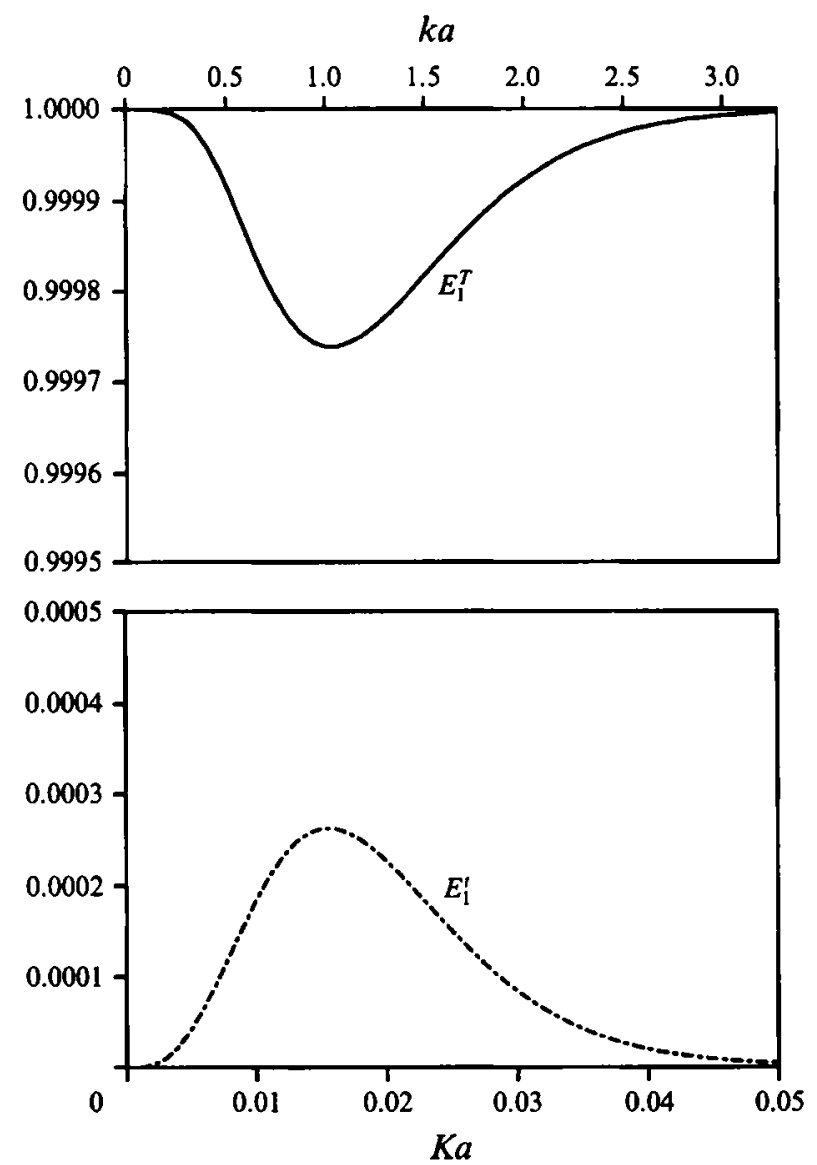

FIGURE 2. Reflected and transmitted energies due a wave of wavenumber $K$ incident on a cylinder in the lower layer; $d / a=2, \zeta / a=-2$ and $\rho=0.97$.

depth evaluated by Thorne (1953). Thus by letting $\rho \rightarrow 0$ in the above analysis we recover the results for the scattering by a horizontal circular cylinder in deep water.

\section{Scattering by a cylinder in the upper fluid}

To develop multipoles singular at $z=\zeta>0$ we require, in addition to the representations (3.1) and (3.2), the following representations valid for $z<\zeta$ :

$$
\begin{aligned}
\frac{\cos n \theta}{r^{n}} & =\frac{1}{(n-1) !} \int_{0}^{\infty} u^{n-1} \mathrm{e}^{u(z-\zeta)} \cos u x \mathrm{~d} u, \\
\frac{\sin n \theta}{r^{n}} & =\frac{1}{(n-1) !} \int_{0}^{\infty} u^{n-1} \mathrm{e}^{u(z-\zeta)} \sin u x \mathrm{~d} u .
\end{aligned}
$$

Suitable multipoles, satisfying the conditions (2.2)-(2.4), are

$$
\phi_{n}^{I s}=\frac{\cos n \theta}{r^{n}}+\frac{1}{(n-1) !} \oint_{0}^{\infty} u^{n-1}\left[A_{U}^{(0)}(u) \mathrm{e}^{u z}+B_{U}^{(0)}(u) \mathrm{e}^{-u z}\right] \cos u x \mathrm{~d} u
$$




$$
\begin{aligned}
\phi_{n}^{I l s} & =\frac{1}{(n-1) !} \oint_{0}^{\infty} u^{n-1} C_{U}^{(0)}(u) \mathrm{e}^{u z} \cos u x \mathrm{~d} u \\
\phi_{n}^{I a} & =\frac{\sin n \theta}{r^{n}}+\frac{1}{(n-1) !} \Psi_{0}^{\infty} u^{n-1}\left[A_{U}^{(1)}(u) \mathrm{e}^{u z}+B_{U}^{(1)}(u) \mathrm{e}^{-u z}\right] \sin u x \mathrm{~d} u \\
\phi_{n}^{I I a} & =\frac{1}{(n-1) !} \oint_{0}^{\infty} u^{n-1} C_{U}^{(1)}(u) \mathrm{e}^{u z} \sin u x \mathrm{~d} u
\end{aligned}
$$

where

$$
\begin{aligned}
& A_{U}^{(q)}(u)=(u+K) \mathrm{e}^{-2 u d}\left[(-1)^{n+q+1}(u-K \sigma) \mathrm{e}^{u \zeta}-(u-K) \mathrm{e}^{-u \zeta}\right] /(u-K) h(u), \\
& B_{U}^{(q)}(u)=\left[(-1)^{n+q+1}(u+K) \mathrm{e}^{u(\zeta-2 d)}-(u-K) \mathrm{e}^{-u \zeta}\right] / h(u), \\
& C_{U}^{(q)}(u)=K(1-\sigma) B_{U}^{(q)}(u) /(u-K) .
\end{aligned}
$$

The polar expansions of these multipoles, valid for $r<\zeta$, are

$$
\begin{aligned}
& \phi_{n}^{s}=\frac{\cos n \theta}{r^{n}}+\sum_{m=0}^{\infty} B_{n m}^{s} r^{m} \cos m \theta \\
& \phi_{n}^{a}=\frac{\sin n \theta}{r^{n}}+\sum_{m=1}^{\infty} B_{n m}^{a} r^{m} \sin m \theta
\end{aligned}
$$

where

$$
\begin{aligned}
& B_{n m}^{s}=\frac{1}{(n-1) ! m !} \oint_{0}^{\infty} u^{n+m-1}\left[(-1)^{m} A_{U}^{(0)}(u) \mathrm{e}^{u \zeta}+B_{U}^{(0)}(u) \mathrm{e}^{-u \zeta}\right] \mathrm{d} u, \\
& B_{n m}^{a}=\frac{1}{(n-1) ! m !} \oint_{0}^{\infty} u^{n+m-1}\left[(-1)^{m+1} A_{U}^{(1)}(u) \mathrm{e}^{u \zeta}+B_{U}^{(1)}(u) \mathrm{e}^{-u \zeta}\right] \mathrm{d} u .
\end{aligned}
$$

We note that unlike the case of multipoles singular in the lower layer, the coefficients in the polar expansions of $\phi_{n}^{s}$ and $\phi_{n}^{a}$ are not the same. The far-field behaviour of these multipoles is given by

$$
\begin{aligned}
& \phi_{n}^{I I s} \sim \frac{\pi \mathrm{i}}{(n-1) !}\left[K^{n-1} R\left(C_{U}^{(0)}: K\right) \mathrm{e}^{ \pm \mathrm{i} K x} \mathrm{e}^{K z}+k^{n-1} R\left(C_{U}^{(0)}: k\right) \mathrm{e}^{ \pm \mathrm{i} k x} \mathrm{e}^{k z}\right] \\
& \phi_{n}^{I l a} \sim \pm \frac{\pi}{(n-1) !}\left[K^{n-1} R\left(C_{U}^{(1)}: K\right) \mathrm{e}^{ \pm \mathrm{i} K x} \mathrm{e}^{K z}+k^{n-1} R\left(C_{U}^{(1)}: k\right) \mathrm{e}^{ \pm i k x} \mathrm{e}^{k z}\right]
\end{aligned}
$$

as $x \rightarrow \pm \infty$.

To solve the scattering problem we again write $\phi$ as in (3.16) and the body boundary condition again implies (3.17). First we consider an incident wave of wavenumber $K$. In this case we obtain the systems of equations

$$
\begin{aligned}
& \alpha_{m}-\sum_{n=1}^{\infty} \alpha_{n} a^{n+m} B_{n m}^{a}=-\mathrm{i} \frac{(-K a)^{m}}{m !} \mathrm{e}^{K \zeta}, \quad m=1,2, \ldots, \\
& \beta_{m}-\sum_{n=1}^{\infty} \beta_{n} a^{n+m} B_{n m}^{s}=\frac{(-K a)^{m}}{m !} \mathrm{e}^{K \zeta}, \quad m=1,2, \ldots
\end{aligned}
$$

When the incident wave has wavenumber $k$ we obtain

$$
\alpha_{m}-\sum_{n=1}^{\infty} \alpha_{n} a^{n+m} B_{n m}^{a}=\frac{\mathrm{i}(k a)^{m}}{K(\sigma-1) m !}\left[(-1)^{m+1}(K \sigma-k) \mathrm{e}^{k \zeta}+(K-k) \mathrm{e}^{-k \zeta}\right], m=1,2, \ldots,
$$




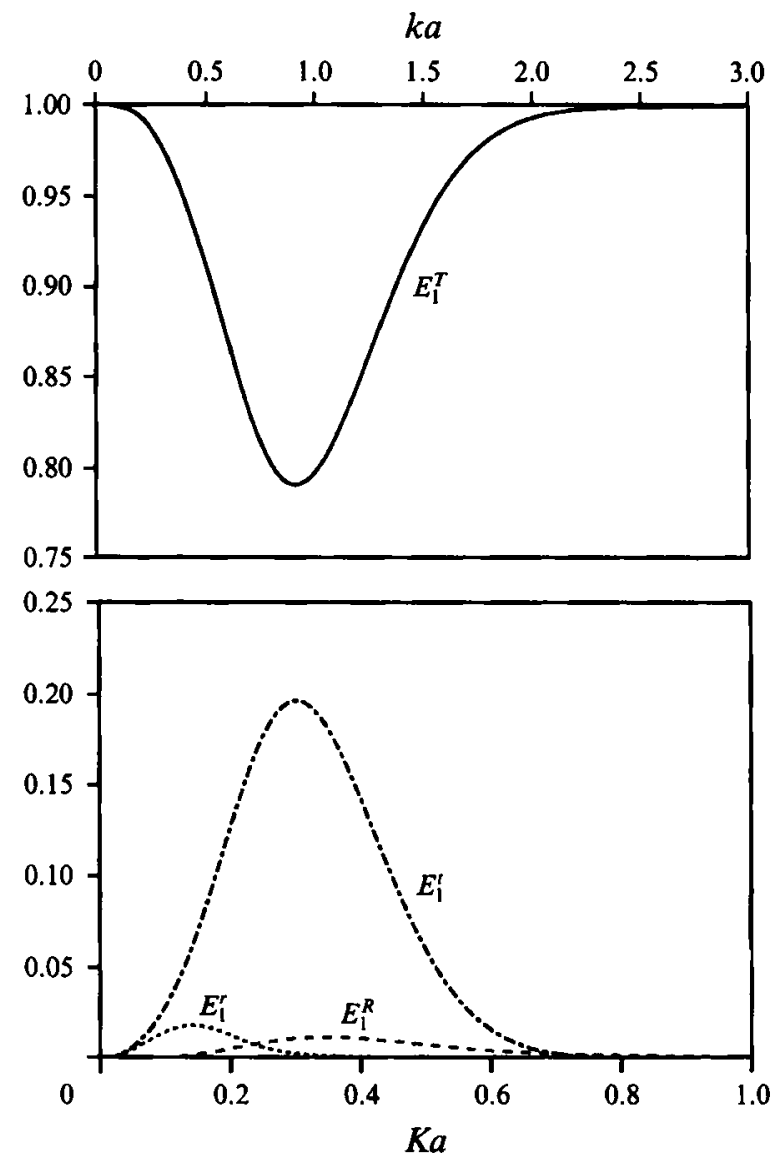

FIGURE 3. Reflected and transmitted energies due a wave of wavenumber $K$ incident on a cylinder in the upper layer; $d / a=2.5, \zeta / a=1.25$ and $\rho=0.5$.

$$
\beta_{m}-\sum_{n=1}^{\infty} \beta_{n} a^{n+m} B_{n m}^{s}=\frac{(k a)^{m}}{K(\sigma-1) m !}\left[(-1)^{m}(K \sigma-k) \mathrm{e}^{k \zeta}+(K-k) \mathrm{e}^{-k \zeta}\right], m=1,2, \ldots
$$

The reflection and transmission coefficients can be obtained from the far-field form of the potential which is

$$
\begin{gathered}
\phi^{I I} \sim \phi_{i n c}^{I I}+\pi \sum_{n=1}^{\infty} \frac{a^{n}}{(n-1) !}\left\{K^{n-1}\left[ \pm \alpha_{n} R\left(C_{U}^{(1)}: K\right)+\mathrm{i} \beta_{n} R\left(C_{U}^{(0)}: K\right)\right] \mathrm{e}^{ \pm \mathrm{i} K x} \mathrm{e}^{K z}\right. \\
\left.+k^{n-1}\left[ \pm \alpha_{n} R\left(C_{U}^{(1)}: k\right)+\mathrm{i} \beta_{n} R\left(C_{U}^{(0)}: k\right)\right] \mathrm{e}^{ \pm \mathrm{i} k x} \mathrm{e}^{k z}\right\}
\end{gathered}
$$

as $x \rightarrow \pm \infty$.

In order to illustrate the conversion of energy from one mode to another we will consider some geometrical parameters unrelated to the case of underwater tube bridges for which the reflected and transmitted energies are considerable. Thus if we take $d / a=2.5, \zeta / a=1.25$ and $\rho=0.5$ then the reflected and transmitted energies due to an incident wave of wavenumber $K$, computed from (4.20) after solving (4.16) and (4.17), are shown in figure 3. Figure 4 shows the results for the same parameters 

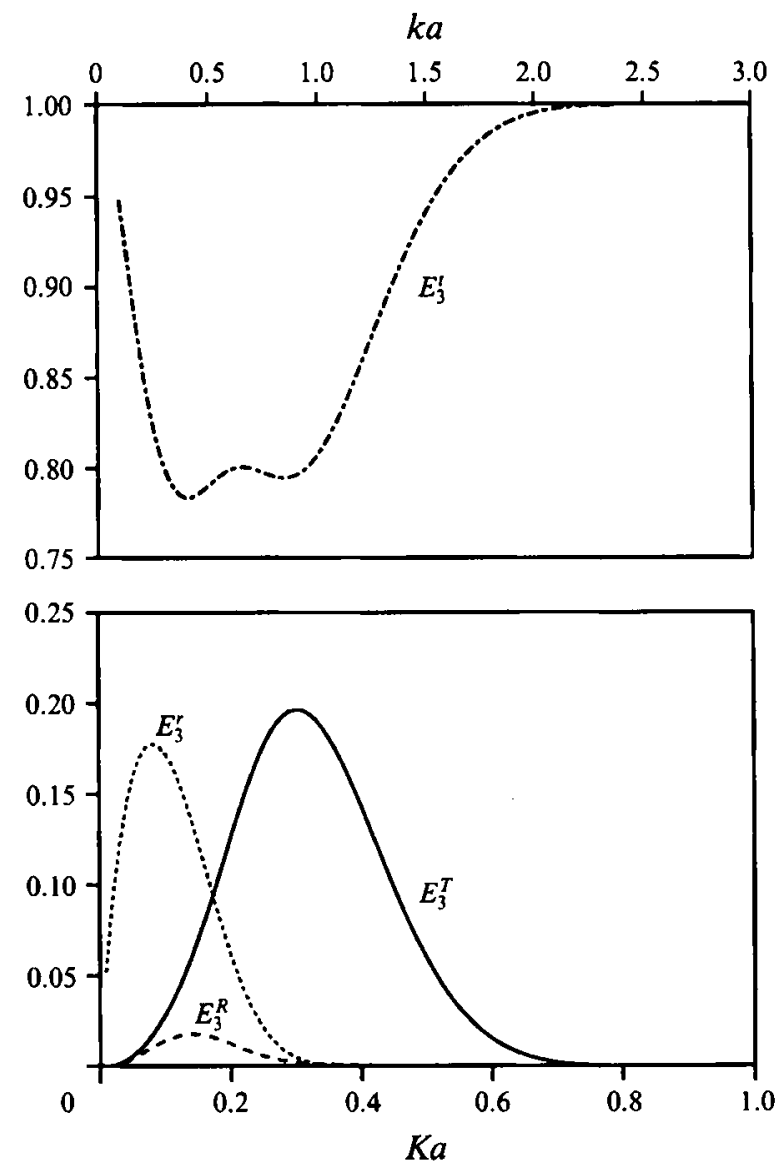

FIGURE 4. Reflected and transmitted energies due a wave of wavenumber $k$ incident on a cylinder in the upper layer; $d / a=2.5, \zeta / a=1.25$ and $\rho=0.5$.

but an incident wave of wavenumber $k$. These were computed by solving (4.18) and (4.19) though of course they could have been obtained from the results in figure 3 by using equations (2.48), (2.49), (2.52) and (2.53). The conservation of energy condition (2.35) shows that for any given value of $K a$ the sum of the values of the four curves in either figure must be unity. The equivalence of $E_{1}^{r}$ and $E_{3}^{T}$ and of $E_{1}^{r}$ and $E_{3}^{R}$ is clear from the figures.

If we let $\rho \rightarrow 0$ in this problem then it can be shown that the multipoles defined by (4.3) and (4.5) go over to the single-layer multipoles for finite depth evaluated by Thorne (1953). Thus by letting $\rho \rightarrow 0$ in the above analysis we recover the results for the scattering by a horizontal circular cylinder in finite water depth.

\section{Conclusion}

In this paper we have examined the relationships that exist between the solutions of two-dimensional radiation and scattering problems in two-layer fluids where the upper fluid is bounded above by a free surface and the lower fluid is infinite in extent. In such a situation propagating waves can exist at two different wavenumbers for any given frequency and we have shown that in the case where a body is symmetric 
about a vertical line the scattering problems involving an incident wave at each of these wavenumbers are related. A systematic derivation, using Green's theorem, of all the reciprocity relations for such problems has been carried out including extensions to the two-fluid case of the Haskind and Newman relations.

We have then considered the scattering problems for two specific geometries, namely a horizontal circular cylinder situated entirely within either the upper or lower layer. These problems have been solved using multipole expansions. In a single-layer fluid of infinite depth it is well-known that no energy is reflected by such a cylinder and we have shown that for a cylinder in the (infinite) lower layer of a two-layer fluid this is again the case, with no energy reflected at either of the possible wavenumbers. The amount of energy that is transferred between wavenumbers in the transmitted energy is also very small.

For a cylinder in the upper layer zero reflection is not observed. This is to be expected since letting the density of the lower layer tend to infinity transforms the problem into a single-layer finite-depth scattering problem for which it is known that non-zero reflection exists.

\section{REFERENCES}

BAINES, P. G. 1984 A unified description of two-layer flow over topography. J. Fluid Mech. 146, 127-168.

Benjamin, T. B. 1967 Internal waves of permanent form in fluids of great depth. J. Fluid Mech. 29, 559-592.

Dean, W. R. 1948 On the reflexion of surface waves by a submerged circular cylinder. Proc. Camb. Phil. Soc. 44, 483-491.

Evans, D. V. \& Linton, C. M. 1989 Active devices for the reduction in wave intensity. Appl. Ocean Res. 11, 26-32.

Fris, A., Grue, J. \& PALM, E. 1991 Application of Fourier transform to the second order 2D wave diffraction problem. In M.P. Tulin's Festschrift: Mathematical Approaches In Hydrodynamics (ed. T. Miloh), pp. 209-227. SIAM.

Gradshteyn, I. S. \& Ryzhix, I. M. 1965 Tables of Integrals, Series and Products. Academic Press.

KasSEM, S. E. 1982 Multipole expansions for two superposed fluids, each of finite depth. Math. Proc. Camb. Phil. Soc. 91, 323-329.

Keulegan, G. H. 1953 Characteristics of internal solitary waves. J. Res. Natl Bur. Stand. 51, 133.

LAMB, H. 1932 Hydrodynamics (6th edn). Cambridge University Press. Reprinted 1993.

LoNG, R. R. 1956 Solitary waves in one- and two-fluid systems. Tellus 8, 460.

Newman, J. N. 1976 The interaction of stationary vessels with regular waves. In Proc. 11th Symp. on Naval Hydrodynamics, London.

Stokes, G. G. 1847 On the theory of oscillatory waves. Trans. Camb. Phil. Soc. 8, 441-455. Reprinted in Mathematical and Physical Papers, Vol. 1, pp. 314-326. Cambridge University Press.

StURova, I. V. 1994 Hydrodynamic forces on a submerged cylinder advancing in waves of twolayer fluids. In Proc. 9th Intl Workshop on Water Waves and Floating Bodies, Kuju, Japan, pp. 199-203.

Thorne, R. C. 1953 Multipole expansions in the theory of surface waves. Proc. Camb. Phil. Soc. 49, 707-716.

URSELL, F. 1950 Surface waves on deep water in the presence of a submerged circular cylinder I. Proc. Camb. Phil. Soc. 46, 141-152.

WU, J.-H. 1992 The second order wave loads on bodies in stratified ocean. In Proc. 7th Intl Workshop on Water Waves and Floating Bodies, Val de Reuil, France, pp. 297-301. 